\title{
EFICIENCIA DEL SECTOR BANCARIO COLOMBIANO Y CHILENO EN LA ADMINISTRACIÓN DE LOS MÁRGENES FINANCIEROS
}

\author{
Nidia Parada Algarra \\ Facultad de Negocios, Gestión y Sostenibilidad \\ Programa de Especialización en Gerencia de Finanzas \\ Especialista en Gerencia de Finanzas \\ nidia_parada@yahoo.es \\ Guillermo Rodríguez Esteves \\ Facultad de Negocios, Gestión y Sostenibilidad \\ Programa de Especialización en Gerencia de Finanzas \\ Especialista en Gerencia de Finanzas \\ guilleroes@hotmail.com
}

\section{Resumen}

El objetivo principal del presente artículo fue, revisar y comparar los márgenes financieros (estos se calculan de acuerdo con la metodología de la Superintendencia Financiera de Colombia, la cual se realiza sobre los activos totales) del sistema bancario colombiano frente a un país referente de la región, para nuestro caso, Chile, con respecto a su evolución en los servicios bancarios a nivel de Latinoamérica. Para este análisis, se utilizó información financiera comparativa de los dos últimos años (2016 y 2017) registrada por los dos países en las páginas web de las superintendencias que vigilan el sector financiero, con el fin de evaluar la eficiencia en la administración de estos márgenes y la incidencia que tiene en el usuario final.

Lo anterior, permitió evidenciar que en Colombia los servicios bancarios, no son los óptimos comparados con el país de referencia, debido a que la estructura de gastos operativos es demasiado elevada respecto a los ingresos que percibe, lo cual conlleva afectar al usuario financiero con los altos costos de endeudamiento, bajos rendimientos en sus ahorros y costos elevados en la administración de sus productos. Por tanto, este tipo de instituciones deben adoptar medidas que permitan optimizar estas erogaciones, y hacer énfasis en lo que se refiere al uso de medios electrónicos para operaciones transaccionales.

Palabras claves: Eficiencia, márgenes financieros, sector bancario, Colombia y Chile. 


\section{Introducción}

De acuerdo con un estudio elaborado por el Banco Mundial (2013), el sector financiero colombiano presenta serios problemas de competitividad y eficiencia si se compara con instituciones del mundo desarrollado e incluso de países similares a Colombia como Argentina y Chile.

Estos problemas se reflejan en el usuario financiero a través de altos costos de endeudamiento, bajos rendimientos en sus ahorros y costos elevados en la administración de sus productos. En este sentido, el presente artículo tiene como objetivo revisar y comparar los márgenes financieros, para el caso del sector financiero se toma la metodología de la Superintendencia Financiera de Colombia, que es dividir cada uno de los rubros y utilidades del estado de resultado y dividirlo en los activos totales, ya que estos son los generadores del ingreso, estos márgenes son como medida de competitividad y eficiencia, del sistema bancario frente a un país referente de la región, para este caso Chile, debido a su evolución en los servicios financieros a nivel de Latinoamérica.

Para lograr el objetivo, se han definido los siguientes objetivos específicos. En primer lugar, identificar la estructura del sistema financiero en los dos países; en segundo lugar, analizar la estructura de márgenes del estado de resultados, y como objetivo final, comparar e identificar la eficiencia de los diferentes márgenes financieros, brindando recomendaciones para subsanar la problemática planteada.

El presente artículo, lo compone cuatro partes, fuera de la presente introducción, una parte, donde se señala la evolución histórica de los sistemas financieros de Colombia y Chile, la segunda la que se refiere a la metodología para realizar la revisión y comparación de los márgenes financieros, por último, el resultado obtenido de dicho análisis, las conclusiones y recomendaciones.

\section{Evolución histórica del Sistema Financiero de Colombia y Chile}

Es importante, en primer lugar, realizar una reseña, evolución, además de conocer la estructura y normatividad de los sistemas financieros en Colombia y Chile. Sobre el particular, se puede mencionar que la evolución del sector financiero en un país está siempre ligada a la de su sociedad y su economía. De ahí que la evolución de los sistemas financieros de estos dos países, responden a la evolución de sus ideas y de sus economías a lo largo de doscientos años de vida de manera independiente (Méndez, 2012).

En sus inicios, tanto en Colombia como en Chile, operaba con rudimentarios esquemas de crédito heredados de la Colonia, los cuales se fueron desarrollando hasta lograr un refinado sistema con el cual opera actualmente. Luego de vivir las experiencias de la banca libre, de la banca centrada en la operación de corto plazo, de la banca de fomento, de la banca estatal y de la banca especializada, logró llegar a la banca universal para el caso de Chile donde 
posee un tipo de banca que tiene como filosofía ofertar todos los productos y servicios a todos los clientes potenciales a nivel mundial (Caballero, 2010).

En Colombia, siguiendo la evolución histórica que hace Caballero (2010), por primera vez surge la idea, sin éxito, de formar un banco estatal en 1821. Años más tarde esta actividad (bancaria) es clasificada como comercial por parte de la normatividad colombiana con lo que surgen los primeros establecimientos bancarios.

Sin embargo, "la banca comercial propiamente dicha se iniciaría en los años sesenta del siglo XIX cuando la economía comenzó a vincularse con el extranjero a través de las exportaciones agrícolas. Se implantó el modelo conocido como de "banca libre" bajo el cual, en ausencia de un banco central y de la regulación estatal” (Caballero, 2010, pág. n.a.).

Por múltiples factores este sistema probó no ser eficiente para el sistema colombiano. De acuerdo con Caballero (2010), luego de muchos intentos finaliza este período de "banca libre" con la creación, en 1923, como resultado de las recomendaciones de la misión Kremmerer, del Banco de la República, como autoridad monetaria y la Superintendencia Bancaria para regular y supervisar al sistema bancario.

El sistema fue evolucionando con la creación del crédito de fomento, fondeado por el banco de la República y posteriormente la aparición de las corporaciones de ahorro y vivienda, como entidades especializadas en la financiación de construcción y compra de vivienda.

De un sistema de especializado como se describió anteriormente, se inicia a finales del siglo pasado una transición al sistema de transición hacia un sistema de banca universal de los bancos comerciales, es decir una misma entidad ofreciendo las diferentes opciones de crédito. "Las crisis financieras - la de los ochenta y la de fin de siglo, la más profunda de todas- dieron lugar a procesos de cierre de entidades y de fusiones y adquisiciones entre ellas, lo cual facilitó la consolidación y el reforzamiento del sistema en los principios del siglo XXI. La apertura a la inversión extranjera en el sector financiero de los años noventa impulsó, igualmente, la transformación del sector financiero en Colombia" (Caballero, 2010, pág. n.a.). teniendo hoy en día un sistema cuya solidez le ha permitido capotear las últimas grandes crisis de manera exitosa y gozar con un alto nivel de confianza por parte del público, pero con grandes retos en materia de inclusión.

Para el caso específico de Chile, la banca ha experimentado numerosos cambios desde la década de los setenta, donde se evidenciaba la ausencia de medios tecnológicos que ocasionaban unos altos márgenes una oferta de productos limitada, escasa competencia, altos márgenes y una profesionalización casi inexistente. En la década de los ochenta, después de superar la crisis financiera de 1982-1984, los profesionales de la tecnología diseñan un procesamiento en batch centralizado que permite el manejo transaccional de carteras masivas y aparecieron los primeros cajeros automáticos. En esta época se presentaron muchas fusiones y adquisiciones de bancos que permitieron una mayor competencia en el sistema financiero, lo cual tuvo como consecuencia la caída de los precios de los productos 
financieros, tanto en términos de tasas de interés como de comisiones, como consecuencia de la reactivación de las economías al bajar los costos de las líneas de financiamiento y generar cambios normativos. Todo esto condujo a que las entidades financieras mejoraran en gran proporción sus eficiencias operaciones a través de automatización de procesos y una gestión más eficiente de la banca. En la década de los noventa se dieron los cambios más importantes como fueron: Alto crecimiento e incrementos en eficiencia, consolidación y concentración del Mercado, integración internacional y aumento de la participación extranjera en la propiedad (Banking News, 2008)

Por otra parte, es importante precisar los marcos normativos de los dos países para establecer sobre qué leyes se respaldan para implementar los costos financieros de los diferentes productos que manejan en el mercado. Para el caso del sistema bancario colombiano, la Constitución Nacional consagra, en su artículo 335, que la actividad financiera, bursátil y aseguradora que en la medida en que implica el manejo, aprovechamiento e inversión de recursos de captación, es una actividad de interés público; por ello, se señala que esta actividad solamente puede ser ejercida con previa autorización del Estado, según lo establezca la ley. Así mismo, establece que, el Gobierno Nacional, al intervenir en esta actividad, debe promover la democratización del crédito. Dicha regulación se enmarca en el Estatuto Orgánico del sistema financiero, el cual aplica para todos los establecimientos bancarios, fondos de pensiones y cesantías, seguros, comisionistas de seguros.

Para el caso de Chile, Las entidades financieras realizan actividades que están definidas dentro del perímetro regulatorio. Los sistemas de fiscalización, para este tipo de entidades, tienen recursos limitados, por lo que la fiscalización, se realiza principalmente sobre las denuncias que se presentan. Por lo anterior, es importante definir la responsabilidad individual de los clientes financieros, utilizando mecanismos como, por ejemplo, informándose sobre si dichas entidades están debidamente registradas como entidades fiscalizadas, pues eso permite reducir el riesgo por pérdidas causadas por fraudes o estafas, así como realizar las consultas respectivas a las entidades fiscalizadores en casos que existan dudas sobre la naturaleza de algún establecimiento bancarios. Evidentemente, el ejercicio de esta responsabilidad y la toma de decisiones de inversión adecuadas se fortalece cuando las personas cuentan con niveles de educación financiera adecuados, por lo que avances en este frente son de mucha importancia (Banco Central de Chile, 2016).

\section{Análisis comparativo de márgenes financieros}

La metodología utilizada para realizar estos análisis fue la elaboración y revisión de datos estadísticos comparativos del sector financiero, es decir, las cifras consolidadas de bancos de Colombia y Chile, de los dos últimos años (2016 y 2017). Para ello se consultaron la información registrada por los dos países en las páginas web de las superintendencias que vigilan el sector financiero. En el caso de Colombia, la Superintendencia Financiera de Colombia y en Chile la Superintendencia de Bancos e Instituciones Financieras de Chile cabe señalar que debido a que los datos estadísticos consultados de los dos países están 
reglamentados bajo normas contables internacionales (NIIF) facilitó el análisis de la información.

Ahora bien, para el cálculo de indicadores y márgenes financieros, se partió de la estructura estándar del estado de resultado de una empresa del sector real, ajustándola al sistema financiero y como denominador se tuvo en cuenta los activos totales consignados en los estados financieros, en el caso del sector financiero se utiliza la metodología de la Superintendencia Financiera de Colombia.

Para ello se consideró que los ingresos financieros son asimilados a las ventas, y están constituidos por toda generación de recursos de la colocación en cartera e inversiones. El costo de venta, para el sector financiero se denomina los gastos financieros, es decir, el costo de los recursos que se requiere para colocar. El resultado entre el ingreso y gasto financiero resulta la utilidad financiera, en otras palabras, la utilidad bruta.

Luego se tiene en cuenta otros ingresos operacionales, que para el caso de los bancos son todos aquellos recursos adicionales que se generan de la operación, por ejemplo, las comisiones de cuota de manejo, comisiones tarjetas de crédito, entre otros. De igual manera, se deduce los gastos administrativos, tanto personales como los generales, que se requieren para poder operar; finalmente, se deduce los gastos por el deterioro de cartera, es decir, la pérdida esperada de la cartera, este resultado genera la utilidad operacional.

Una vez estructurada esta información, se realiza para el caso de este análisis, un neto entre los ingresos y egresos no operacionales, que son todos aquellos recursos que se generan o gastan y que no tiene que ver con la operación del negocio. Por ejemplo, la venta de los bienes recibido en pago, multas y sanciones, venta de activos fijos, entre otros. Esta cifra se suma o resta de la utilidad operacional, lo que genera la utilidad antes de impuesto, a ésta se le resta los impuestos, lo que finalmente resulta la utilidad neta del negocio.

Para obtener los indicadores y márgenes financieros, se tomó el estado de resultados en la estructura anteriormente mencionada y se dividió cada rubro en los activos totales. A continuación, se presenta el resultado de los cálculos realizados. 


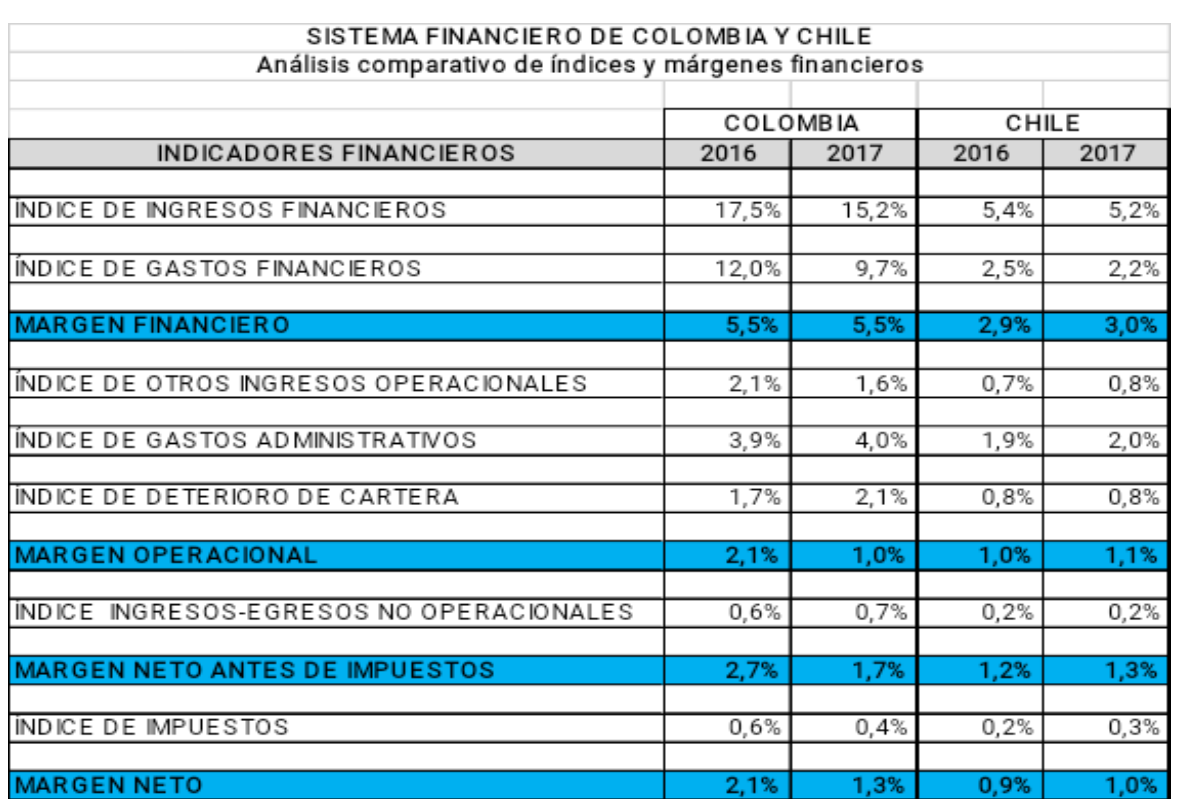

Fuente: Elaboración propia con base en datos de la Superintendencia Financiera de Colombia y de la Superintendencia de Bancos e Instituciones Financieras.

Como se puede observar, el margen financiero del consolidado de los bancos en Colombia para los años 2016 y 2017 fue de 5.5\%, muy superior al de Chile que para el año 2016 fue de $2.9 \%$ y para el 2017 se ubicó en el 3.0\%, al mirar la composición del margen se puede concluir en principio que las tasas de colocación son altas para el caso de Colombia.

Ahora, si se revisa el índice de otros ingresos operacionales, en Colombia para el año 2016, se ubicó en el $2.1 \%$ y se redujo para el año 2017 al 1.6\%. Esta reducción se generó principalmente, porque algunos bancos exoneraron cobros de servicio para ser más competitivos, lo que generó unos indicadores muy por encima a los que presenta Chile para los dos años, $0,7 \%$ y $0.8 \%$ respectivamente, lo que se concluye que los costos de las cuotas de manejo, las comisiones, entre otros cobros, son muy altos para el caso colombiano.

Continuando con el análisis, el índice de gastos administrativos, el Sistema Financiero Colombiano presenta para el año 2016 un indicador del 3.9\% y para el 2017 el $4.0 \%$, que frente a los de Chile, que se ubicaron en $1,9 \%$ y $2.0 \%$ respectivamente, se aprecia una mejor eficiencia en la administración de los recursos que utilizan los bancos en Chile para su operación que los de Colombia.

Por otra parte, es importante analizar el indicador de deterioro de cartera, el cual refleja el nivel de provisiones que se debe realizar con el fin de cubrir el riesgo de crédito (Superintendencia de Bancos e Instituciones Financieras, 2018), para el caso de Colombia en el año 2016 se ubicó en $1.7 \%$ y para el 2017 presentó un crecimiento, situándolo en $2.1 \%$, debido principalmente al aumento de la cartera vencida que tuvo el país en dicho año. Para el sistema financiero de Chile este indicador es de $0.80 \%$ para los dos años, lo que demuestra un mejor comportamiento de la cartera de créditos en dicho país. 
Del análisis anterior, se deduce que el margen operacional para los dos países se ubica alrededor del 1.0\%, excepto para Colombia en el año 2017 que fue del $2.1 \%$ originado principalmente por los otros ingresos operacionales. Es decir, para el último año el margen operacional está en los mismos niveles para los dos sistemas financieros. Lo que permite concluir, que Colombia puede soportar la ineficiencia de los gastos administrativos, gracias al alto ingreso de la colocación y a los otros ingresos operacionales, que afectan de manera directa el flujo y los resultados de los usuarios financieros.

Ahora, en cuanto al margen neto para los dos países en el último año se ubica alrededor del $1.0 \%$, donde se resaltar que en el caso de Colombia los otros ingresos-egresos son mayores que los ingresos-egresos de Chile, pero los impuestos igualmente son ligeramente más altos, lo que a la final generan un margen muy similar.

Por último, del análisis se puede resaltar que, para el caso colombiano como consecuencia de la falta de eficiencia en la administración de los recursos, las tasas de los créditos, tanto para el segmento de personas naturales como para las personas jurídicas son altas, lo que puede afectar el crecimiento. Igualmente, como consecuencia de esta ineficiencia, los costos de los servicios bancarios son altos, lo que también produce unos menores ingresos tanto para las personas como para las empresas.

De otra parte, las inversiones y los ahorros también se ven afectados por las bajas tasas de interés que los bancos ofrecen, ya que los bajos márgenes no le permiten tener mayores rendimientos.

Para terminar, es importante resaltar que la administración de los márgenes financieros del sector bancario, igualmente tienen un impacto importante en la profundización financiera de los países, es así, que Chile para el año 2016 el indicador era del 88\%, mientras que en Colombia se situaba en el 45\% (Caballero, C._2010). 


\section{Conclusiones}

Del análisis del capítulo anterior, permite evidenciar que en Colombia los márgenes no son los óptimos comparados con el país de referencia, debido a que la estructura de gastos operativos es demasiado elevada respecto a los ingresos que percibe, lo cual conlleva afectar al usuario financiero con los altos costos de endeudamiento, bajos rendimientos en sus ahorros y costos elevados en la administración de sus productos.

Este análisis, permite hacer una recomendación al sistema bancario colombiano para adoptar medidas que permitan optimizar estos gastos, especialmente en lo que se refiere a la sistematización de las operaciones transaccionales. Toda vez que en la banca colombiana se siguen realizando un número significativo de operaciones presenciales a través de oficinas o sucursales, que tiene unos costos de operación más altos frente a canales como internet, cajeros automáticos, entre otros, lo que podría disminuir las tasas de colocación y reducir los cobros de servicio y de esta manera favorecer a los usuarios.

Igualmente, si se toman las medidas anteriores podría fomentar la profundización financiera, lo que permite un mejor desarrollo para el país. 


\section{Referencias}

Argáez, C. C. (2015). ¿Qué Transformaciones Ha Vivido El Sector Financiero A Lo Largo De Su Historia? Bogotá: Revista Dinero.

Caballero, C. (2010). Un Rápido Recorrido Por La Historia Del Sector Financiero En Colombia.

Astrid Martínez Ortiz, Luis Alberto Zuleta, Martha Misas, Lino Jaramillo. (2016). La Competencia Y La Eficiencia En La Banca Colombiana. Bogotá D.C.: Fedesarrollo Y Asobancaria.

Banco Central De Chile. (2016). Fundamentos De La Regulación Financiera Y Definición. Santiago De Chile: Banco Central De Chile.

Banco Central De Chile. (2016). Fundamentos De La Regulación Financiera Y Definición. Santiago De Chile: Banco Central De Chile.

Banco Mundial. (2013). Capacidades Financieras En Colombia: Resultados De La Encuesta Nacional Sobre Comportamientos, Actitudes Y Conocimientos Financieros. Bogota.

Banking News. (2008). Breve Historia De Los Bancos En Chile. Banking News.

Carlos Caballero Argáez. (2010). Un Rápido Recorrido Por La Historia Del Sector Financiero En Colombia. Dinero.

Claudia Carvajal. (2018). Las "Lagunas” De La Nueva Ley De Bancos. Diario Uchile.

(2016). Estudio Del Sistema Financiero Chileno. Santiago De Chile: Superintendencia De Bancos E Instituciones Financieras.

Flores Yarela, Walts, David. (2012). El Trimestre Económico, Vol. Lxxix (4), Núm. 316. En El Trimestre Económico, Vol. Lxxix (4) (Pág. 40). Mexico Df: Fondo De Cultura Económica.

Hernan Torlaso, D. D. (2017). Chile: Industria Bancaria Y La Capacidad De Cambiar Con Agilidad. America Retail.

Jose Dario Uribe. (2013). El Sistema Financiero Colombiano: Estructura Y Evolución Reciente. Revista Del Banco De La República, 13.

Méndez, J. P. (2012). Estudio Comparativo De Algunos Sistemas Financieros En América Latina No. 12.

(2017). Panorama Bancario. Santiago De Chile: Superintendencia De Bancos E Instituciones Financieras Chile.

Superintendencia De Bancos E Instituciones Financiera Chile. (2017). Informe Del Desempeño Del Sistema Bancario Y Cooperativas A febrero De 2017. Santiago De Chile: Superintendencia De Bancos E Instituciones Financiera Chile.

Superintendencia De Bancos E Instituciones Financieras De Chile. (2016 Y 2017). Estados Financieros. Santiago De Chile: Superintendencia De Bancos E Instituciones Financieras.

Superintendencia De Bancos E Instituciones Financieras. (2017). Normas Sobre La Gestión Y Posición De Liquidez. Santiago De Chile: Superintendencia De Bancos E Instituciones Financieras.

Superintendencia De Bancos E Instituciones Financieras. (2018). Indicadores Financieros. Santiago De Chile: Superintendencia De Bancos E Instituciones Financieras.

Superintendencia Financiera De Colombia. (2018). Informe De La Actualidad Del Sistema Financiero Colombiano. Bogotá: Https://Www.Superfinanciera.Gov.Co/Publicacion/Informe-Actualidad-Del-SistemaFinanciero-Colombiano.

Superintendencia De Bancos E Instituciones Financieras. (2016 Y 2017). Indicadores. Santiago De Chile: Superintendencia De Bancos E Instituciones Financieras. 